\title{
Investigation of Interphase Effects in Silica-Polystyrene Nanocomposites Based on a Novel Hybrid MD-FE Simulation Framework
}

\author{
Sebastian Pfaller, Gunnar Possart, and Paul Steinmann \\ Chair of Applied Mechanics, Friedrich-Alexander-Universität Erlangen-Nürnberg, \\ Egerlandstraße 5, D-91058 Erlangen, Germany* \\ Mohammad Rahimi \\ Institute for Molecular Engineering, \\ University of Chicago, Chicago, Illinois 60637, $U S A^{\dagger}$ \\ Florian Müller-Plathe and Michael C. Böhm \\ Eduard-Zintl-Institut für Anorganische und Physikalische Chemie \\ and Centre of Smart Interfaces, Technische Universität Darmstadt, \\ Alarich-Weiss-Str. 4, D-64287 Darmstadt, Germany
}




\begin{abstract}
A recently developed hybrid method is employed to study the mechanical behavior of silica - polystyrene nanocomposites (NCs) under uniaxial elongation. The hybrid method couples a particle domain to a continuum domain. The region of physical interest, i.e. the interphase around a nanoparticle (NP), is treated at molecular resolution, while the surrounding elastic continuum is handled with a finite element approach. In the present paper we analyze the polymer behavior in the neighborhood of one or two nanoparticle(s) at molecular resolution. The coarse grained hybrid method allows us to simulate a large polymer matrix region surrounding the nanoparticles. We consider NCs with dilute concentration of NPs embedded in an atactic polystyrene matrix formed by 300 chains with 200 monomer beads. The overall orientation of polymer segments relative to the deformation direction is determined in the neighborhood of the nanoparticle to investigate the polymer response to this perturbation. Calculations of strain-like quantities give insight into the deformation behavior of a system with two NPs and show that the applied strain and the nanoparticle distance have significant influence on the deformation behavior. Finally, we investigate to what extent a continuum-based description may account for the specific effects occurring in the interphase between the polymer matrix and the NPs.
\end{abstract}

Keywords: hybrid method, nanocomposites, interphase, mechanical deformation, silica-polystyrene

\footnotetext{
* sebastian.pfaller@ltm.uni-erlangen.de

$\dagger$ mrahimi@uchicago.edu
} 


\section{INTRODUCTION AND OUTLINE}

Reinforcement of polymers with nanoparticles (NPs), leading to so-called nanocomposites (NCs), is an effective method to improve their mechanical behavior for industrial purposes. The properties of these nanocomposites can be tuned by varying the NP size from the micro- to the nano-scale at the same volume fraction $[1,2]$. The large contact areas between the NP surfaces and the polymer matrix have increased the interest in the region near the NP surfaces, the so-called interphase. Polymer properties in the interphase region differ from those in the polymer bulk [3-7]. These local variations are the main reason for the unique behavior of NCs which cannot be estimated additively from the polymer and NP properties [1]. The thickness estimated for the interphase region, which is usually in the $\mathrm{nm}$ range, depends both on the $\mathrm{NC}$ components and on the quantities used as probes in such analyses, e.g. density, stress, bond orientation, or monomer mobility [8]. Various computational methods have been adopted to investigate NCs at different length scales [911]. However, most of these approaches have limitations and weaknesses when studying the interphase region. Thus, powerful techniques to analyze polymer properties in this region are still required to deepen our understanding of the advanced material properties of nanocomposites. A lack of simulation tools is clearly seen when studying the response of the interphase region to a mechanical deformation.

In the last two to three decades, molecular dynamics (MD) simulations have grown to a powerful tool in the study of polymer properties. However, in spite of the rapid development of computers, simulations of polymeric systems with intermediate or high molecular weights are still impossible at the atomistic scale. For NC materials these limitations are even more serious than for pure polymers, since the size of the NP is often larger than the accessible length scale of atomistic simulations. In order to overcome this problem, multiscale simulation approaches are required. These methods link or combine different resolution levels to access multiple time and length scales. Using different simulation methods and combination strategies, various multiscale simulation approaches have been developed [1216]. In the sequential approaches, simulations at different scales are performed separately, and physical properties (structures, energies, forces, etc.) are transferred from higher to lower resolution. For instance, one can switch from high to low resolution by mapping a group of atoms into a superatom (bead) to reach the mesoscopic time and length scales needed. 
Reducing the number of degrees of freedom due to lumping a group of atoms into a superatom makes it possible to study phenomena like self-assembly and entanglements, which happen only for systems with high molecular weights $[17,18]$. However, switching between different resolutions requires that information details from the high resolution are sacrificed to reach the multiple time and length scales. In contrast to the sequential approaches, hybrid schemes combine the advantages of methods at different length scales by performing simulations at different resolutions simultaneously. They are particularly useful under conditions where detailed information is important only in a restricted region, such as in NC interphases.

Many hybrid methods have been developed to couple different resolutions for different kinds of materials [19-26]. In these schemes, the simulation box is usually divided into two domains with different resolutions. The main challenge in all hybrid methods is the conservation of continuous physical quantities (density, momentum, and geometrical properties) and their fluxes (for hydrodynamic problems) at the interface between two domains. This can be done by exchanging the boundary conditions in the region where the two domains have an overlap. In that region, the levels of resolution as well as the physical quantities of a system smoothly change from one domain to the other. Recently, we have developed a new hybrid molecular dynamics-continuum mechanics method for polymers [27]. In our model, the molecular dynamics domain is treated at a coarse-grained (CG) scale [16] whereas a finite element (FE) method is employed to describe the surrounding continuum. It is clear that this setup prevents the application of periodic boundary conditions (PBCs). To couple the two regions, we have adopted stochastic boundary conditions (SBCs) [28] which force the polymer in the MD domain to show bulk-like behavior. Time-averaged physical quantities from the MD domain are provided to the FE domain via the overlap region. The hybrid method has been tested recently by simulations of pure polystyrene (PS). It has been validated by comparing reaction forces and the Cauchy stress derived by the hybrid setup to a pure FE reference system [27].

Hybrid schemes are not only appropriate for systems where detailed information is important in particular spatial regions. They are also suitable to study multiscale phenomena like deformation and failure $[29,30]$. In fact, these processes are multiscale phenomena which occur over wide time and length scales. In the work at hand, we use our hybrid method in CG resolution [27] to study the mechanical properties of nanocomposite materials formed by atactic PS and silica NPs. In this method, which will be addressed in detail in Section II, the 
system is decomposed into an MD as well as an FE domain. The NP(s) and the interphase region are treated with MD at CG resolution, while the polymer bulk is modeled with FE. The MD domain is in the center of the simulation box is surrounded by the FE domain. With this technique, we can dramatically reduce the system's degrees of freedom without loosing information in the interphase region. The studied sample is deformed by prescribing displacements to the outermost finite element boundaries. A staggered algorithm, which is explained below, is used to transfer information between the FE and the MD domains across the boundary region. After having deformed the hybrid system completely, the behavior of the polymer is studied in the neighborhood of the NPs.

Similar to some of our recent bare MD investigations of nanocomposites [31-34], the present work is focused on silica-polystyrene NCs. We have analyzed NCs with one or two nanoparticles embedded in an atactic PS matrix of 300 chains defined by 200 monomers. In comparison to our recent work [32-34], larger bulk regions can be considered in the present hybrid study. A second advantage is the accurate treatment of load steps; for a recent CG based deformation approach see [31]. In Section IV, we validate our hybrid method by investigating the response of a pure PS system and that of a NC with a single NP to a spatial deformation. For this purpose, we perform simulations with one ungrafted silica NP located in the center of the MD domain. Additionally, we compare the stress-strain curves obtained from the hybrid method and from pure MD simulations. In a next step, we investigate the averaged orientation of local polymer segments to figure out the polymer response to the presence of the NP, which has structural implications and is thus not accurately accessible by continuum mechanics.

Beyond this, we concentrate in Section V on NCs with two NPs embedded into a comparatively large polymer matrix. Such a setup frequently arises when NPs locally agglomerate in NCs. In our analysis we have considered both agglomerated model structures as well as ones without allowing close NP-NP contacts. Due to the large number of polymer chains to be considered, such systems with realistic NP diameters of tens of nanometers cannot be captured any more by pure particle approaches. Instead, we replace the polymer matrix located far away from the NPs by an FE description and thus show the capability of our hybrid method for practically relevant purposes. 


\section{HYBRID METHOD}

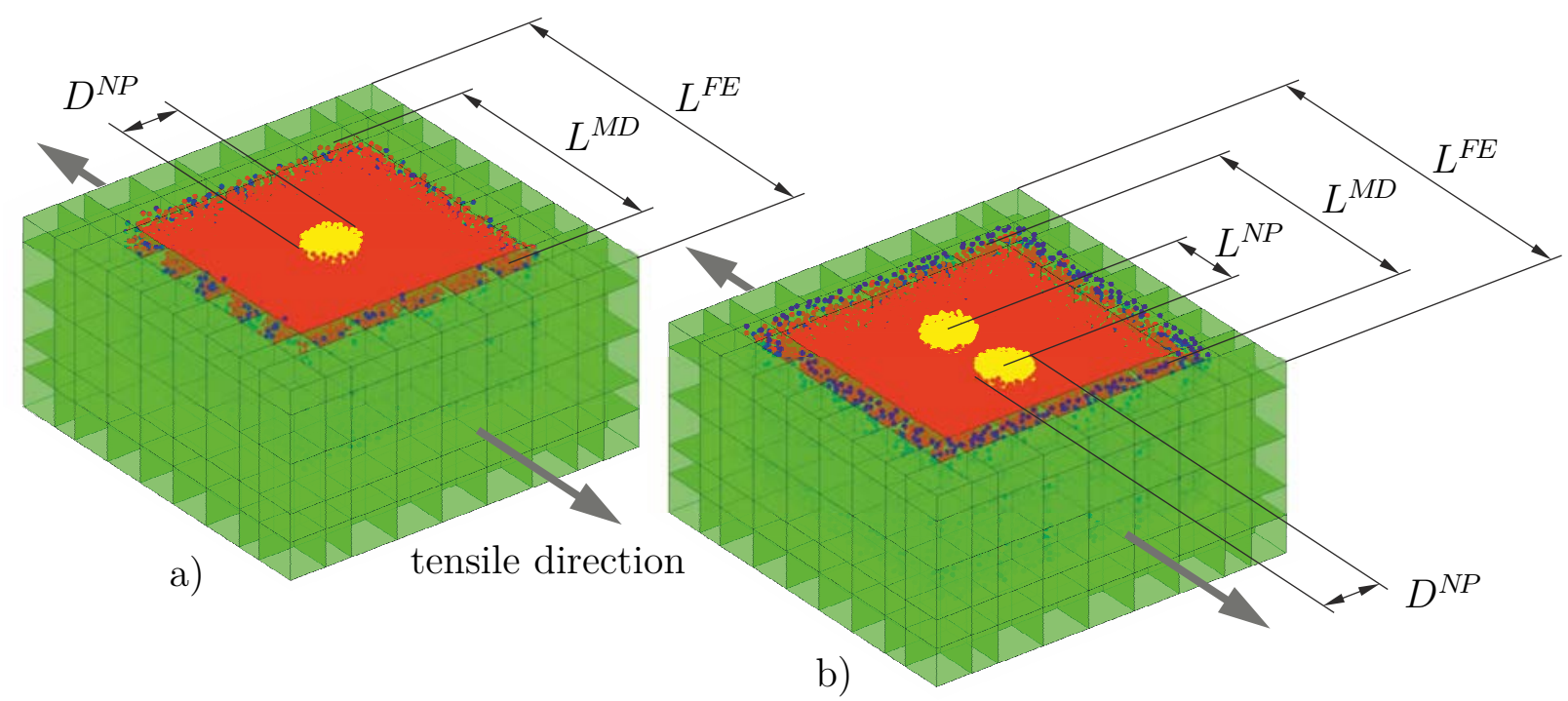

FIG. 1. Simulated hybrid systems in the undeformed configurations; only the bottom halves are displayed: a) one single silica nanoparticle (yellow), b) two silica nanoparticles (yellow) embedded in a polystyrene matrix and simulated using the hybrid scheme (finite elements green, anchor points blue, polystyrene superatoms red); geometric quantities such as nanoparticle diameter and domain extensions are introduced in Section IIIC

The mechanical properties of silica-PS nanocomposites are studied using our recently developed hybrid method. The theoretical and technical details of this approach can be found elsewhere [27]; here we just give a brief explanation of its basic principles. The MD domain, treated by a coarse grained model [32], is coupled to the FE domain. It is located in the center of the simulation box and surrounded by the FE region. Both domains overlap in a boundary region. As mentioned in the introduction, the main challenge in hybrid simulations is the definition of suitable boundary conditions between the two domains. They have to conserve physical quantities at the interfaces. We have adopted non-periodic boundary conditions, so-called stochastic boundary conditions (SBCs), which satisfy this condition [28]. In addition, strain and stress are transferred between the domains across the boundary region by using auxiliary or virtual particles, so-called anchor points. Figure 1 displays the bottom halves of the hybrid simulation boxes with either a single NP or two NPs. The CG beads of the NPs are depicted as yellow spheres, whereas those of the polymer 
matrix are red spheres. The anchor points in the boundary region are symbolized as blue spheres and the finite elements are given in transparent green. To confine the polymer inside the MD domain, the chains in the boundary region are connected to the anchor points by springs. The positions of these artificial auxiliary entities inside the boundary region remain fixed during the MD simulation. Acting as an external potential for the MD region, the ensemble of anchor points exerts a pressure to define a thermodynamic state inside it. To reduce the influence of the anchor points on the dynamics of the PS beads, dissipativeparticle dynamics (DPD) is employed in the boundary region to introduce some randomness in the aforementioned dynamics $[35,36]$. As a result, DPD pretends that there is a bulk of polymer chains beyond the boundary region and, therefore, imposes that the polymer in the outer MD domain behaves like the bulk. To summarize, the anchor points as constituents of the chosen SBCs have the following functions: i) they provide the information transfer between the MD and the continuum domain; ii) they suppress (reduce) the probability that polymer beads leave the MD domain; iii) thus they conserve approximately the pressure and enable the definition of a thermodynamic state.

The anchor points are tethered to the surrounding FE domain by means of the Arlequin method, a coupling approach developed by Ben Dhia and Rateau [37]. Originally, this technique has been designed, among others, to couple static FE and particle domains. Both regions overlap in a so-called bridging domain, which coincides with the boundary region in our method introduced above. Therein, a weighting factor $\alpha$ blends the total energies of both descriptions and an additional kinematic coupling constraint ensures matching of continuum and particle domain displacements. The minimization of the total energy of the hybrid system under consideration of the kinematic constraint leads to an optimization problem that is solved using Lagrange multipliers. Here, we apply the Arlequin framework only to the coupling between the anchor points and the FE domain, whereas the coupling between the anchor points and the MD region is realized by a widely employed approximation in MD, i.e. a harmonic potential, see Figure 2. This degree of sophistication has been used also in former atomistic simulations [33] serving as a reference for the coarse-graining [31, 32]. The entire system has to be solved by a staggered algorithm which is based on our preliminary, one-dimensional considerations of such approaches [38].

Eventually, two sub-problems are computed iteratively: 

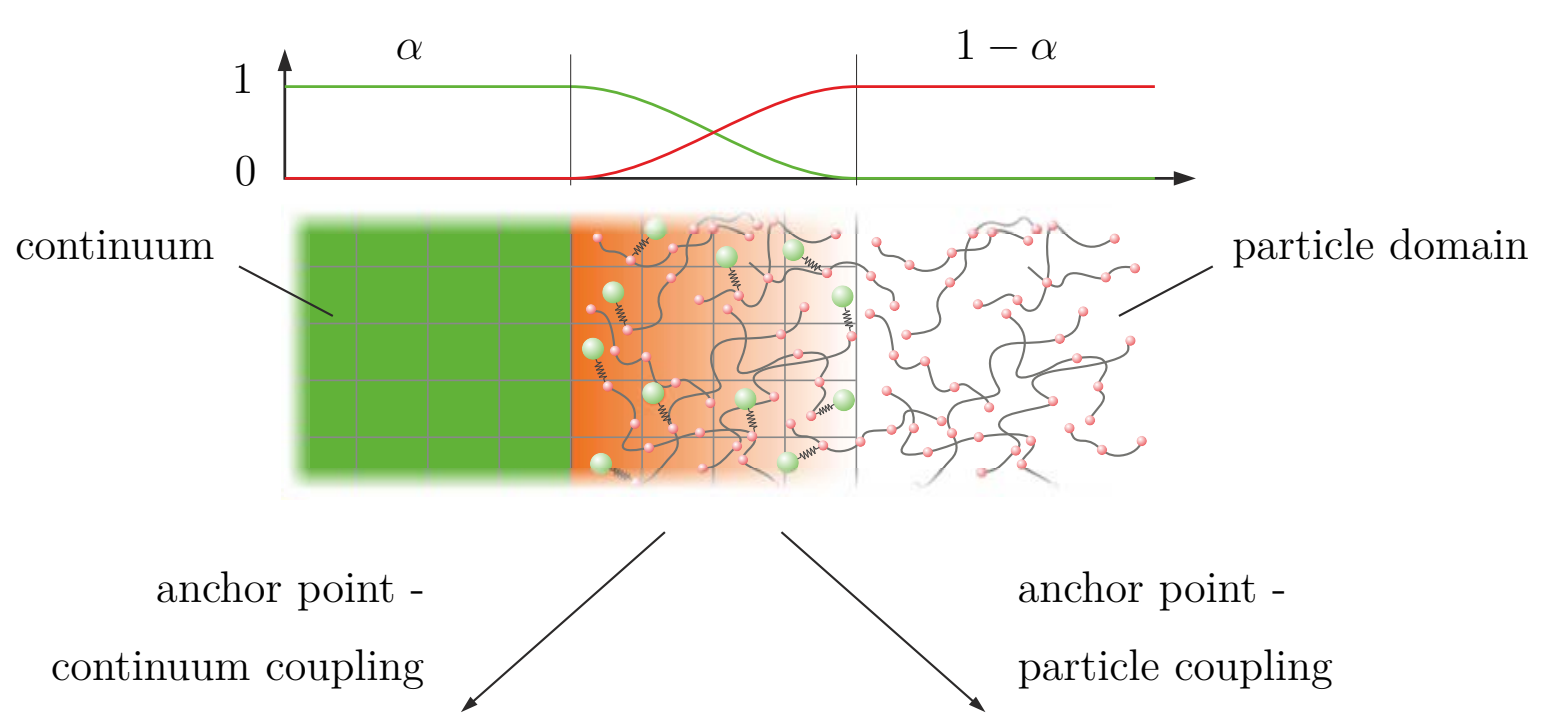

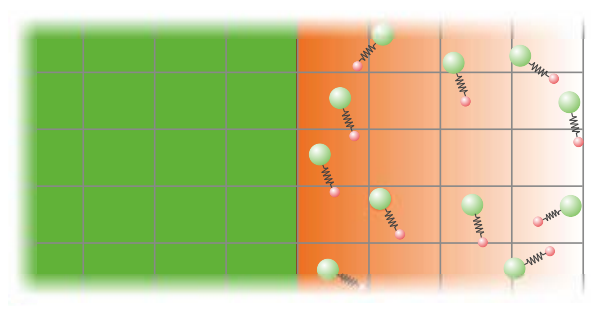

via Arlequin coupling

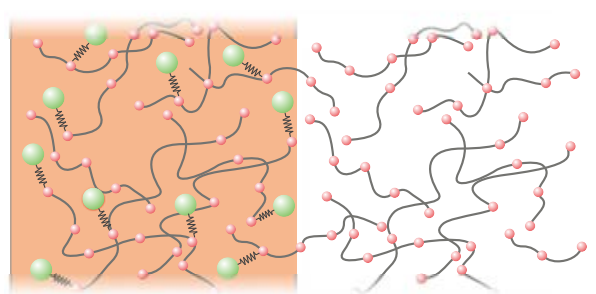

via harmonic springs and SBC

FIG. 2. Spatial set-up of the coupled system: MD particles are given as small spheres, anchor points as large spheres; the overall coupling consists of a coupling between the anchor points and the continuum (via the Arlequin method) and of a coupling between the anchor points and the polymer beads; the weighting factor $\alpha$ is used for energy blending

1. The MD system is simulated under SBCs using discrete time steps. The time-averages of forces exerted on the anchor points by the MD superatoms serve as input data for the subsequent FE step.

2. The FE domain coupled to the anchor points achieves an intermediate optimum by applying the Newton-Raphson algorithm. After this step, updated anchor point positions are transferred to the subsequent MD simulation and another iteration loop starts from step 1.

This scheme as depicted in Figure 3 is continued until the hybrid system converges. The simulation overhead resulting from the consideration of the FE domain and the MD-FE 


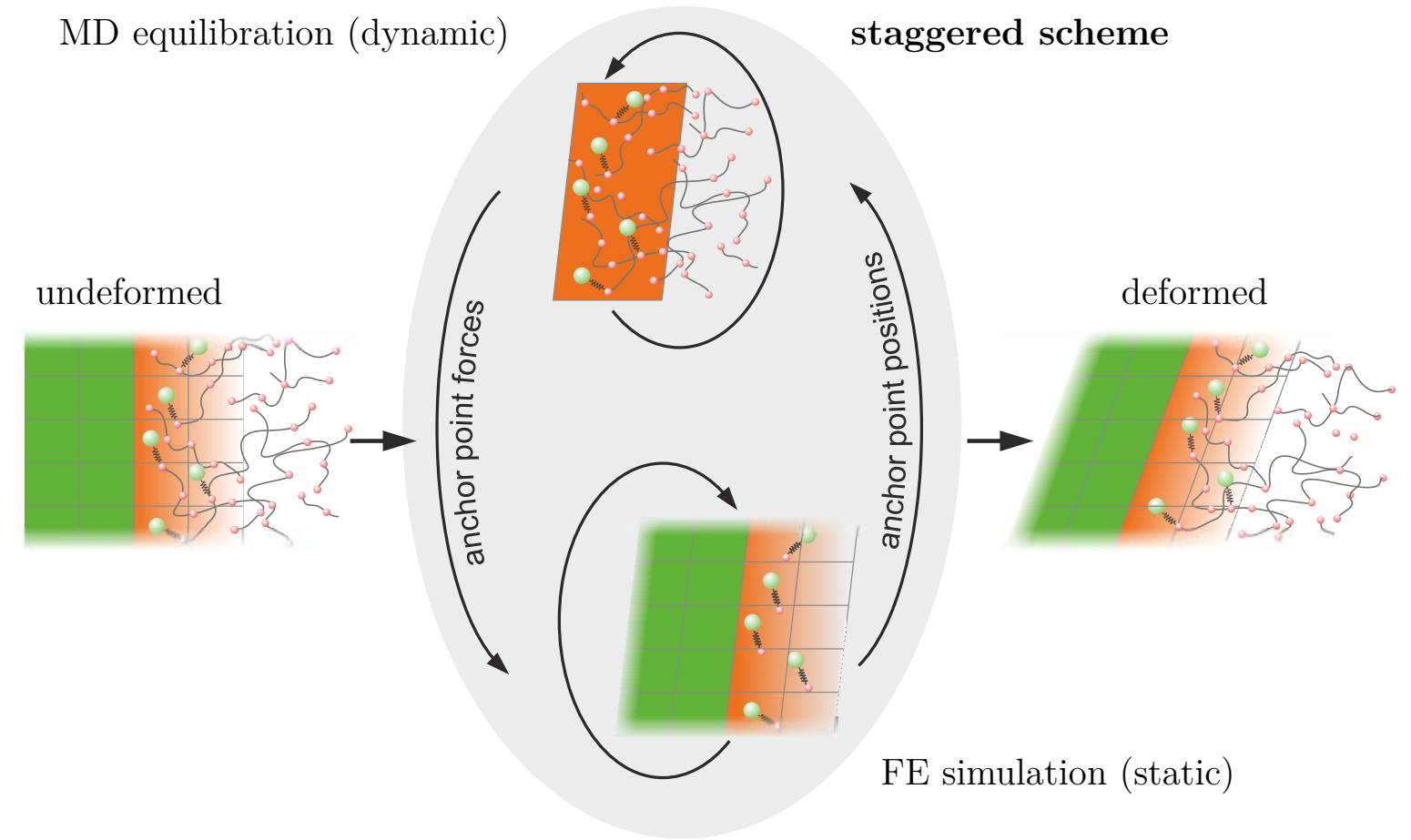

FIG. 3. Departing from the undeformed configuration, the staggered coupling scheme iterates between a static FE simulation (coupling between anchor points and the continuum) and an equilibration of the MD system (coupling between anchor points and the particle domain) until equilibrium of the deformed system is obtained

coupling is small compared to a bare MD system of comparable domain size, i.e. in the range of a few percent. The hybrid method is, of course, much more expensive than a bare MD simulation due to the staggered solution algorithm. It roughly requires the time of a bare MD equilibration multiplied by the number of MD-FE iteration cycles. Strategies to reduce the computational cost are currently investigated. Note, however, that the comparison is only hypothetical as the system sizes accessible by the hybrid method significantly exceed those which can be considered by bare MD. Details on this procedure can be found elsewhere [27]. There, we have also demonstrated the capability and accuracy of the hybrid method for atactic PS under uniaxial tension. Furthermore, some preliminary MD-FE results for silicaPS NCs have been presented, whereby a uniaxial strain up to $7 \%$ has been applied to the simulation box. The difference between the Cauchy stress obtained in a pure FE reference system and in the hybrid simulation has been analyzed. In contrast to pure FE models, the stress in the hybrid method is not perfectly homogeneous. We have shown that the 
polydispersity in the boundary region caused by the adopted SBCs generates these stress deviations. They are largest in the vicinity of edges and corners of the boundary region, whereas they almost vanish elsewhere. Later we will come back to this topic which is caused by the nonperiodic system setup. The robustness of our approach has been demonstrated in a recent uncertainty quantification analysis [39]. We have shown that modifications of the model parameters do not have a critical influence on quantities of interest as long as they are chosen in a realistic range.

In our hybrid scheme, any external loads can be applied via boundary conditions to the FE domain, which makes it a well suited tool for investigating stress and strain distributions in e.g. NCs. In contrast, it is rather difficult to apply arbitrary loads to conventional MD systems under PBCs. To apply uniaxial strain, the length of a pure MD simulation box has to be increased into one direction and the positions of particles have to be scaled to create reliable starting positions for MD. Although this works well in case of homogeneous materials, undesired effects may occur for inhomogeneous materials as e.g. NCs. Here, as we have verified recently [8], NPs generate stress anisotropies in their neighborhood. This makes it difficult in pure MD systems to locally analyze strain and compare it with the total strain to distinguish soft and stiff polymer regions.

\section{SYSTEM SETUP}

\section{A. MD domain}

As mentioned in former sections, we have analyzed silica-polystyrene NCs with one or two NPs. The chosen silica NPs have a radius of $2 \mathrm{~nm}$ and are defined by $873 \mathrm{SiO}_{2}$ beads. Their centers are located at the positions of the $\mathrm{Si}$ atoms in a $\mathrm{SiO}_{2}$ crystallite. The initial PS matrix contains 300 chains with 200 monomers (molecular weight $\approx 2000$ a.m.u.), i.e. the initial polymer sample is monodisperse. This monodispersity is slightly violated under the influence of the adopted (stochastic) boundary conditions. In a recent uncertainty analysis, however, we have shown that the deviations from monodispersity in the inner MD core are small $(<$ $2 \%$ ) [39]. In the CG scheme proposed by Qian et al. [40] for the PS matrix each PS monomer is represented by one CG bead whose interaction center is located in the center of mass. Two different beads ( $R$ and $S$ ) are required to take into account the chirality of atactic PS. 
Detailed information on the currently adopted CG force field and the atomistic force field for the simulation of an underlying reference state have been given in our recent studies [32, 33]. In order to derive a CG force field by iterative Boltzmann inversion (IBI) [41], an atomistic force field considering all bonded and nonbonded interactions as well as atomic net charges is required for preceding atomistic reference calculations. For pure PS the OPLS all-atom force field [42] has been chosen in our atomistic study of silica-PS nanocomposites [33]. Silica Lennard-Jones and Coulomb elements have been taken from a force field for bulk crystalline silica [43]. All nonbonded interactions in PS and the studied NCs have been evaluated by the Lorentz-Berthelot mixing rule [44]. Additional improvements of the original atomistic force field, e.g. Kirkwood approximation, are documented in our first atomistic study of silicaPS systems [33]. The CG force field adopted in the present hybrid simulation is the one described in [31, 32]. The IBI based CG potentials describe the nonbonded intramolecular and intermolecular interactions as well as the bonded ones between two neighboring bonded beads. For three neighboring beads an angular potentials is taken into account while the torsional potential is neglected in the CG picture, for details see [33]. To allow hybrid simulations as a function of the separation between between two NP we have set the NPNP interaction equal to zero even if the NP separation is smaller than the cutoff. Thus their interaction is indirect, i.e. through the polymer chains. The undisturbed radius of gyration of the PS chains amounts to $3.4 \mathrm{~nm}$ which is of the same order of magnitude as the silica diameter. The accuracy of the adopted CG scheme has been tested recently [32] by comparing radial distribution functions and radial profiles of monomer number densities in atomistic and CG resolution. A theoretical method to generate all initial configurations has also been described in our CG precursor study [32]. At first, the NPs are placed to predefined positions in the simulation box. Then, a self-avoiding random walk is employed to generate polymer chains around the NPs. In order to equilibrate the initial configuration, the systems are simulated for $20 \mathrm{~ns}$ under NPT conditions until they reach the desired density $\left(\sim 970 \mathrm{~kg} \mathrm{~m}^{-3}\right)$ in the PBC framework. All MD runs are carried out at a pressure of $101.3 \mathrm{kPa}$ and a temperature of $590 \mathrm{~K}$ with a time step of $5 \mathrm{fs}$ and a $1.5 \mathrm{~nm}$ cutoff. A Berendsen thermostat (with a coupling time of $0.2 \mathrm{ps}$ ) and a barostat (with a coupling time of $5 \mathrm{ps}$ and an isothermal compressibility of $1.0 \times 10^{-6} \mathrm{kPa}$ ) are used to control temperature and pressure. After equilibration, all systems are cooled at a constant rate of $10 \mathrm{~K} \mathrm{~ns}^{-1}$ to a temperature of $100 \mathrm{~K}$, i.e. a value below the glass transition $T_{g}(170 \mathrm{~K}$ for PS in the employed 
CG potential [31]). After that, the boundary conditions of all systems are switched from periodic to stochastic. Details about this procedure have been explained elsewhere [28]. Our motivation to perform hybrid simulations below $T_{g}$ is to eventually study phenomena such as fracture or damage in the context of polymer nanocomposites. These processes are irrelevant in the melt state. In this context we want to point out that $T_{g}$ depends on the adopted resolution. The CG value is much lower than the atomistic $T_{g}$ value of $368 \mathrm{~K}$ [33]. Physical reasons for this difference have been touched in [45] where it has been emphasized that $T_{g}$ does not only depend on the resolution of a simulation but also on the details of the chemistry. The thickness of the boundary region is roughly $2.0 \mathrm{~nm}$ and the anchor points are distributed randomly in this region, but their density is decaying exponentially towards the MD domain. They are connected to the polymer chains via harmonic springs with a force constant $k=1400 \mathrm{~kJ} \mathrm{~mol}^{-1} \mathrm{~nm}^{-2}$. The force constant connecting bonded PS beads is of the same order of magnitude [28]. For the DPD equations of motion, a friction coefficient of $10 \mathrm{pN} \mathrm{ps} \mathrm{nm}^{-1}$ is used. Dissipative-particle dynamics also acts as a thermostat in the boundary region to keep the temperature of the whole MD domain constant at $100 \mathrm{~K}$.

\section{B. Coupling to the FE domain}

The boundary region is inserted into a cubic FE domain with an outer edge length of $30 \mathrm{~nm}$. Due to the flexibility of our hybrid method, this setup can be employed for systems with one and two NPs. More details on the FE domain and its coupling to the MD region may be found in our previous paper [27]. The employed setup is depicted in Figure 1 for both system types.

Before applying external loads, an initial equilibration of the hybrid system has to be carried out. For this purpose, the Young's modulus $E$ in the FE domain is chosen very low $(E=0.1 \mathrm{MPa})$, while Poisson's ratio is set to zero. We have performed $300 \mathrm{MD}-\mathrm{FE}$ iterations to fully equilibrate the hybrid box and to reduce the pressure inside the MD domain to zero. In each iteration step, an MD simulation of $0.5 \mathrm{~ns}$ is performed.

After this initial equilibration, the systems are subjected to uniaxial tension by prescribing uniform displacements at two opposite surfaces of the FE domain, see Figure 1. The FE region is now treated as a linear elastic domain with Young's modulus $E=800 \mathrm{MPa}$ and Poisson's ratio $\nu=0.3$. Both parameters have been identified from an MD simulation of PS 
under PBC [31] and tested in one of our previous studies [27].

In the following, one or two silica NPs are placed into the MD region of the hybrid system. With this setup, individual NPs can be simulated in a comparatively large polymer matrix as it is frequently the case for nanocomposites with low weight or volume contents of NPs.

\section{Data evaluation}

Figure 1 displays the undeformed configurations of the hybrid systems and the associated geometric quantities. In the following, upper case letters refer to the undeformed initial configuration, whereas lower case letters indicate the deformed state. The geometric quantities used for the evaluation of the simulation results are given in Figure 1 and read:

NP diameter:

$$
\begin{array}{r}
D^{N P} \\
L^{N P}, \ell^{N P}
\end{array}
$$

center-to-center distance between the two NPs:

distance between the respective mean positions of anchor points in

two opposite MD boundaries perpendicular to the tensile direction:

$L^{M D}, \ell^{M D}$

overall length of the entire system (i.e. MD and FE domains):

$L^{F E}, \ell^{F E}$

Based on these definitions, integral strain measures, which describe the relative change of displacements, may be introduced as follows:

$$
\begin{array}{ll}
\text { integral strain of NP centers: } & \varepsilon^{N P}=\frac{\ell^{N P}-L^{N P}}{L^{N P}} \\
\text { integral MD-box strain: } & \varepsilon^{M D}=\frac{\ell^{M D}-L^{M D}}{L^{M D}} \\
\text { integral FE-box strain: } & \varepsilon^{F E}=\frac{\ell^{F E}-L^{F E}}{L^{F E}} \\
\text { normalized strain of NP centers: } & \tilde{\varepsilon}^{N P}=\frac{\varepsilon^{N P}}{\varepsilon^{M D}}
\end{array}
$$

To obtain the mean positions of anchor points defining the current MD box size, we consider anchor points located within a square of $13 \mathrm{~nm} \times 13 \mathrm{~nm}$ at the center of the boundary domain. 


\section{METHOD VALIDATION: PS WITH ONE NANOPARTICLE}

In this section we employ a single ungrafted silica NP initially placed in the center of the simulation box. During the simulations, the NP is allowed to move with the polymer matrix, i.e. it is not artificially fixed to the domain center. As mentioned above, the chosen stochastic boundary conditions generate polydispersity in the boundary region, but only a minor one in the core region near the NP [28, 39]. Figure 1 a) illustrates the hybrid box for the system with a single silica NP. Since the distance from the NP surface to the boundary region is $6.5 \mathrm{~nm}$ and thus relatively large, the $\mathrm{FE}$ domain as well as the outer MD domain reproduce pure PS bulk properties. For the FE domain, parameters (Young's modulus $E=800 \mathrm{MPa}$ and Poisson's ratio $\nu=0.3$ ) obtained from MD simulations of pure PS are employed [27]. The total number of anchor points used to confine the MD region is 8947. In analogy to other parameters, this value cannot be justified on the basis of physical principles. But we have shown that such a number is a fair compromise between the reduction of the polymer dynamics and the hindrance of polymer bead transitions from the MD to the FE region [28]. Note that, due to the polydispersity caused by the SBC procedure, the density of anchor points is not spatially constant. In the corners of the boundary region it is higher than in the central regions where a roughly constant averaged density of approximately 2.1 anchor points per $\mathrm{nm}^{3}$ is attained. The size of the boundary domain is related to the cut-off radius chosen for the non-bonded interactions of the MD domain. In general, if the size is too small, the superatoms in the pure MD domain loose interaction partners and thus will be exposed partly to a vacuum. Criteria to fit parameters such as the extension of the coupling region or the polymer-anchor point force constant have been studied in connection with an uncertainty quantification [39].

The tensile strain $\varepsilon^{F E}$ is applied within 8 loads steps of $1 \%$ each. Every load step consists of 100 MD-FE iterations, each of which comprises 100000 MD time steps. In Figure 4 a), we compare the averaged FE stress $\bar{\sigma}_{N C}^{F E}$ of the NC in tensile direction to that of a pure PS system $\bar{\sigma}_{P S}^{F E}$ investigated in our previous publication [27]. Both stress averages are based on the stress values of the FE nodes at the surfaces perpendicular to the tensile direction.

In addition, we introduce the relative difference between both stresses as

$$
\delta \bar{\sigma}^{F E}=\frac{\bar{\sigma}_{N C}^{F E}-\bar{\sigma}_{P S}^{F E}}{\bar{\sigma}_{P S}^{F E}}
$$



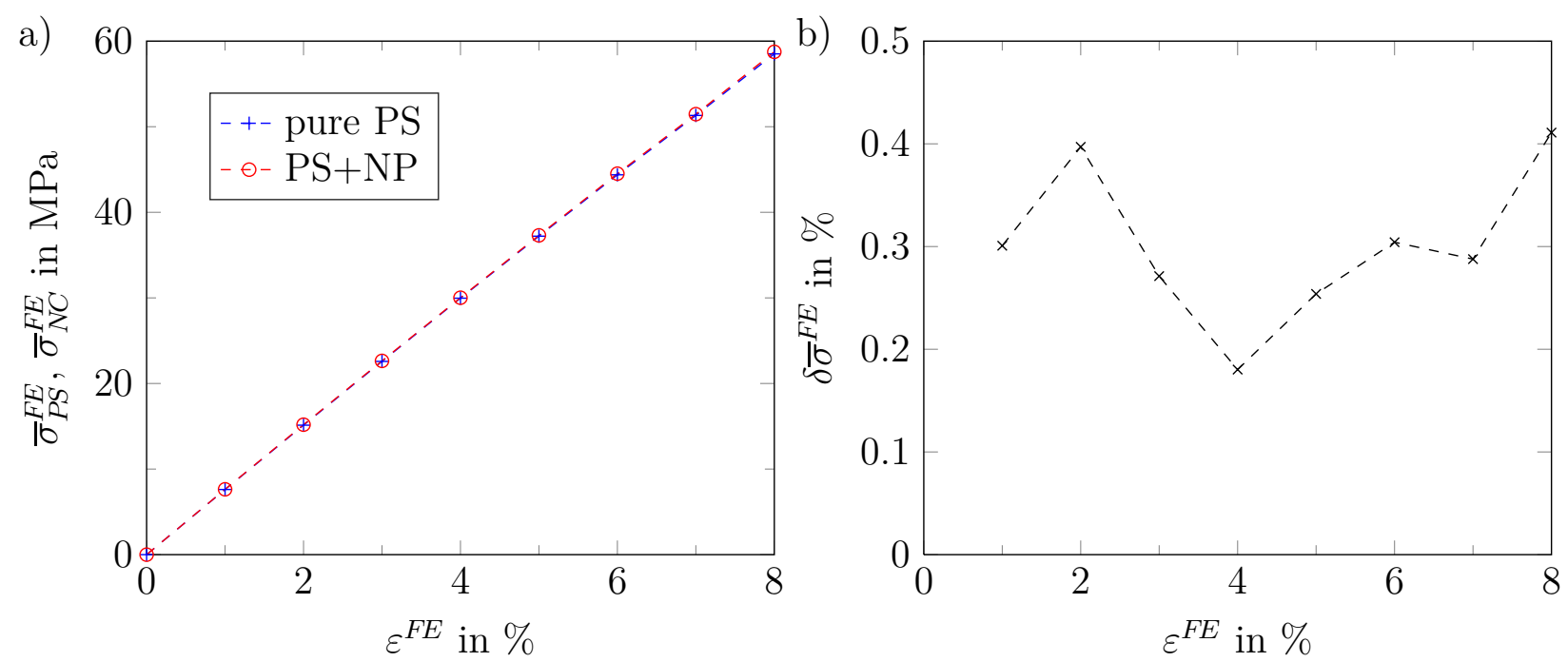

FIG. 4. a) Mean FE stress $\bar{\sigma}_{P S}^{F E}$ (pure PS) and $\bar{\sigma}_{N C}^{F E}$ (NC with a single NP) evaluated after 100 MD-FE iteration steps in each load step; b) relative difference $\delta \bar{\sigma}^{F E}$ at each load step; a strain of $\varepsilon^{F E}=1 \%$ is applied in every load step; both figures have been published previously [46]

Due to the stiff, but small NP, a slightly stiffer response of the NC has to be expected. As evident from Figure $4 \mathrm{~b}$ ), our hybrid method is able to capture even this minor influence properly and is thus well-suited to investigate particle effects in NCs with low NP contents.

In order to investigate in detail the NC's response to the deformation, the orientation of polymer segments with respect to the deformation direction is calculated. Again, we apply uniaxial tension step-wise via increasing the tensile strain $\varepsilon^{F E}$ of the FE domain. Segments of five monomers are chosen to examine the orientation; they are small enough to monitor the polymer structure on a local scale. The orientation of a segment is computed by the second-order Legendre polynomial $P_{2}$, given by

$$
P_{2}=<\frac{3}{2} \cos ^{2} \theta-\frac{1}{2}>
$$

where $\theta$ is the angle between the deformation direction and a vector joining PS beads $i$ and $i+4$. Note that this definition differs from our recent one where we have measured the orientation between the longest axis of the squared gyration tensor and the surface normal [33]. In contrast to this individual chain descriptor, we have now considered a global one related to the deformation direction. The symbol $<\bullet>$ denotes ensemble averaging. For a system of randomly oriented polymer segments $P_{2}=0$ holds, for perfect parallel orientation $P_{2}$ equals 1 , and for an orthogonal segment arrangement $P_{2}$ amounts to -0.5 . 


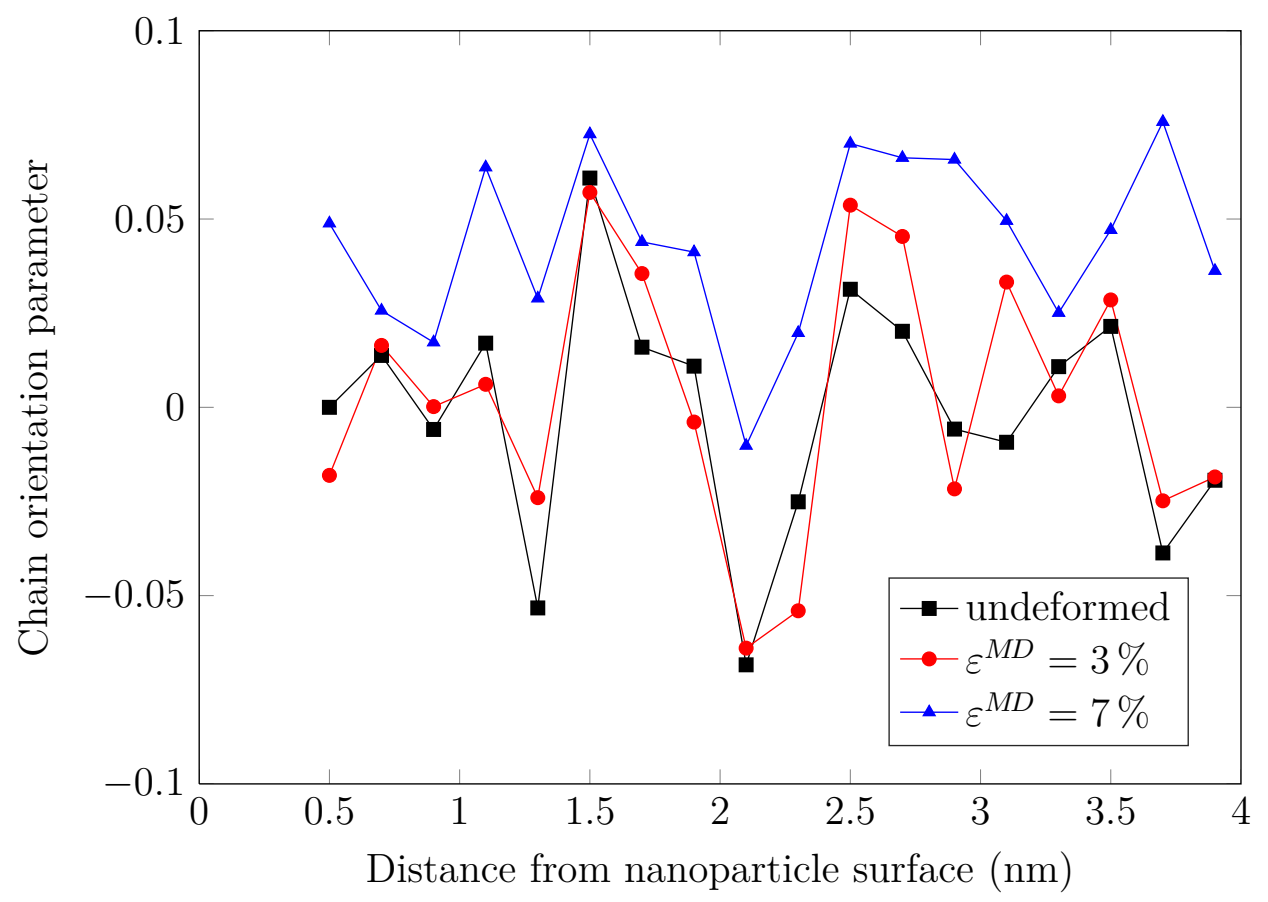

FIG. 5. Order parameter $P_{2}$ of chain segments consisting of five monomers, displayed as a function of the distance from the NP surface in case of the undeformed sample and for strains of $3 \%$ and $7 \%$

Figure 5 shows the orientation parameter as a function of the distance from the NP surface for the integral MD-box strain $\varepsilon^{M D}$ between $0 \%$ and $7 \%$. The position of bead $i+2$ is taken to calculate the distance between the segment and the NP surface. The order parameter $P_{2}$ is averaged over all segments within spherical bins of thickness $0.2 \mathrm{~nm}$ around the NP. For the undeformed system, the direction of the polymer segments relative to the deformation direction is random. Therefore $P_{2}$ fluctuates around 0 and has a mean value of -0.001 , which is very close to zero. If strain is applied, the polymer chains are expected to rotate toward the direction of the deformation and $P_{2}$ should increase. However, up to approximately $3 \%$ strain, there is neither a significant change in the distance dependency of $P_{2}$ nor in the mean value, which increases only slightly to 0.002 , i. e. the polymer chains roughly keep their orientation. In case of larger strains, the polymer chains rotate and align considerably in loading direction, which is reflected by increased values of $P_{2}$. At $7 \%$ strain, $P_{2}$ reaches a maximum mean value of 0.043 . Thus, we are able to verify by our simulation that, as one would expect, the grade of chain orientation increases with increasing strain. On the other hand, the low maximum values for $P_{2}$ obtained in the present work are not surprising due to 
the limited mobility of polymer chains in the glassy state as considered here. Furthermore, the increase of the orientation parameter obviously does not depend on the distance from the NP surface. This differs from the distance dependence observed for the polymer orientation relative to the NP surface normal [33]. In the present case the polymer chains remain almost randomly oriented, irrespective of their location with respect to the NP, but slightly align when the system is exposed to uniaxial tension. Due to the brittleness of polystyrene below the glass transition temperature, investigations for strains larger than $7 \%$ could not be carried out with the adopted hybrid method. First steps to allow simulations for larger deformations have been done.

Concluding this section, we may state that our hybrid method allows for detailed, particlebased investigations in the vicinity of the NP for a system with a comparatively large volume of the polymer matrix. The results show that even slight changes in the system's response and configuration due to the presence of an NP or due to the magnitude of the applied strain can be captured and evaluated properly. These are essential demands to simulation methods especially with regard to NCs with low volume or mass contents of NPs. In a next step, we focus on systems with two NPs and study their effect on the deformation of the system.

\section{INTERPHASES IN NCS: PS WITH TWO NANOPARTICLES}

In the following we perform uniaxial tension tests on systems with two NPs at different initial distances. As a result of NP-NP interactions of zero, agglomeration is prevented allowing hybrid simulations as a function of the nanoparticle separation. We present the normalized strain $\tilde{\varepsilon}^{N P}$ of NP centers as introduced in Figure 4 for five different samples with initial surface distances between the NPs of $1.93 \mathrm{~nm}, 3.14 \mathrm{~nm}, 4.44 \mathrm{~nm}, 4.87 \mathrm{~nm}$, and $5.47 \mathrm{~nm}$. Based on an NP diameter of $4 \mathrm{~nm}$, the corresponding initial center-to-center distances between the NPs $L^{N P}$ are $5.93 \mathrm{~nm}, 7.14 \mathrm{~nm}, 8.44 \mathrm{~nm}, 8.87 \mathrm{~nm}$, and $9.47 \mathrm{~nm}$, respectively. In the first part of this section, we perform simulations employing our hybrid method, whereas we focus on a structurally identical pure FE setup in the second part for comparison. After that, we discuss the results and compare the capabilities of the hybrid method and pure FE simulations. 


\section{A. Coupled MD-FE system}

As above, the coarse-grained MD simulations are performed at $100 \mathrm{~K}$ with the same anchor point force constant and size of the boundary region. The MD systems are prepared such that the centers-of-mass of the NPs are located along the deformation axis at a predefined distance. During the equilibration and cooling procedure, the centers-of-mass of the two NPs are restrained to the deformation axis. Thus, the NPs can move only in one direction.

The number of anchor points in all systems is now approximately 11000 ; a schematic of the simulation box is given in Figure 1 b). In each load step, the system is deformed by $0.5 \%$ and equilibrated for $50 \mathrm{MD}-\mathrm{FE}$ iteration steps. MD simulations of $0.5 \mathrm{~ns}$ are performed in each iteration step, such that the deformation rate is the same as in the case of a single NP.

As hybrid simulations are very time-consuming, we restrict the analysis of the normalized strain $\tilde{\varepsilon}^{N P}$ between NP centers to a single initial configuration for each NP distance, a computational setup leading to some statistical fluctuations. For the discussion of general trends, however, the observed uncertainties are considered acceptable. Hybrid simulations of nanocomposites with a broad spectrum in NP number and size are postponed to later contributions.

Figure 6 shows the normalized strain $\tilde{\varepsilon}^{N P}$ between NP centers as a function of the global strain $\varepsilon^{M D}$. For homogeneous materials, $\varepsilon^{N P}$ and $\varepsilon^{M D}$ should be identical leading to $\tilde{\varepsilon}^{N P}=1$. Such a behavior cannot be expected in NCs characterized by different physical properties of the $\mathrm{NP}(\mathrm{s})$, the interphase, and the polymer bulk. Local modifications due to the interphase decay with the distance to the NP $[31,32,47]$. Therefore, spatial inhomogeneities can be enhanced furthermore by NP agglomerations crucially influencing the material properties of NCs [48]. It is generally believed that these agglomerations contribute to the mechanical fragility of NCs [48]. This problem and other issues arising in the context of NCs have been also investigated by bare MD simulations [17, 49-51]. From Figure 6 we obtain that $\tilde{\varepsilon}^{N P}$ tends to increase with increasing $\varepsilon^{M D}$, whereby the system with the smallest NP distance renders the lowest values for $\tilde{\varepsilon}^{N P}$. In general, $\tilde{\varepsilon}^{N P}$ is below one for small $\varepsilon^{M D}$ and achieves values around one and slightly above for larger values of $\varepsilon^{M D}$. This implies that the centers of the NPs do not follow the overall strain $\varepsilon^{M D}$ for small $\varepsilon^{M D}$, i. e. the NPs "stick together" to a certain extend. On the other hand, $\varepsilon^{N P}$ and $\varepsilon^{M D}$ are almost equal for large $\varepsilon^{M D}$ and thus 


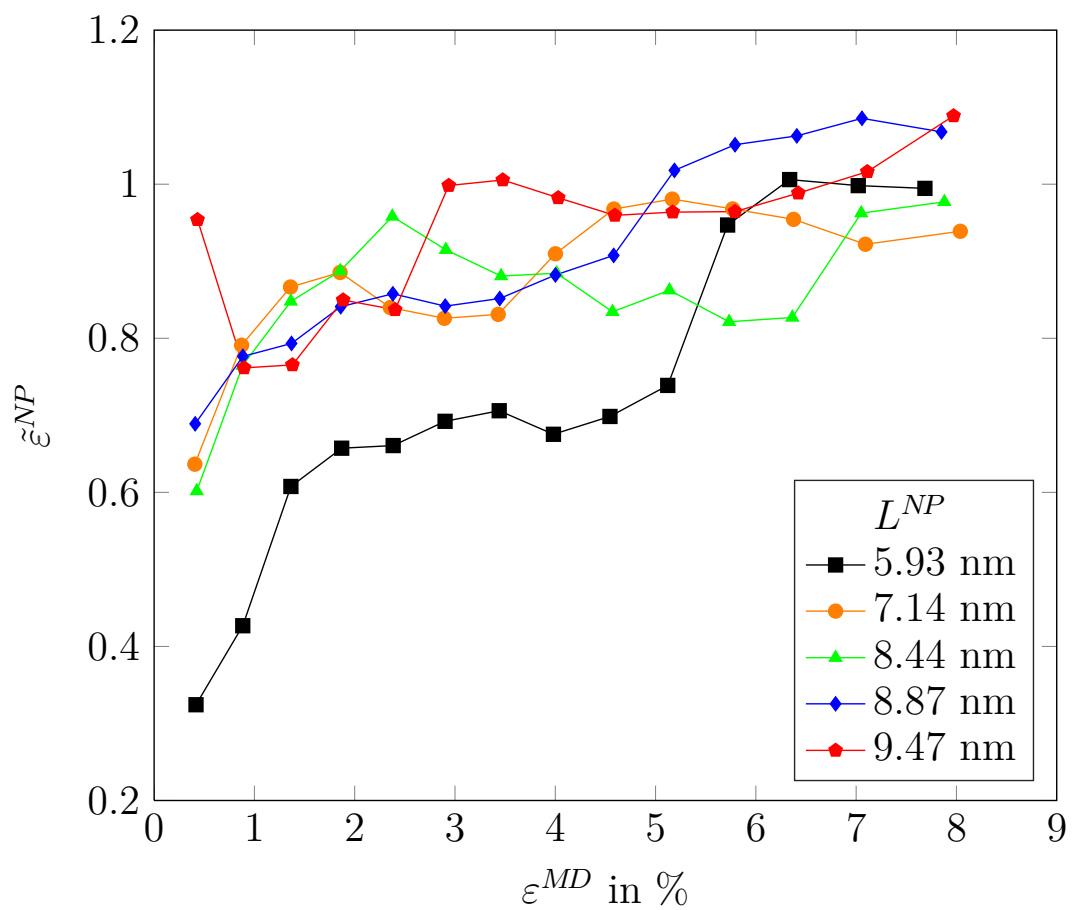

FIG. 6. Hybrid simulation: normalized strain $\tilde{\varepsilon}^{N P}$ between NP centers as a function of global strain $\varepsilon^{M D}$ for different initial interparticle distances $L^{N P}$

the NPs move according to the overall strain. Despite the aforementioned fluctuations in the simulation results it seems to be allowed to point out that $\tilde{\varepsilon}^{N P}$ is by far the smallest for the sample with minimum initial NP distance. We subsequently propose possible explanations for these observations.

\section{B. Pure FE system}

In order to evaluate our findings from the hybrid MD-FE simulations, we investigate a pure FE representation of the hybrid simulation box. For this comparison to a continuum mechanics description, we have to consider carefully the interaction between the NPs and the polymer matrix. From a continuum mechanics point of view and in contrast to an MD consideration, it is possible to define a purely geometric coupling between the NPs and polymer matrix by tethering the surface of each NP to the polymer matrix. In such a setup, neither the mechanical properties of the polymer matrix nor of the NPs are affected by the presence of NPs or the polymer and thus, in terms of continuum mechanics, no polymer-NP interphases occur. As we show below, strain inhomogeneities in this case appear only due 
to the geometric setup of regions with different mechanical properties. Thus, we refer to these effects in the following as "geometric effects". In contrast, if the material properties of the polymer matrix would have to be altered as a function of the distance to the NP to describe the system properly, interphases have to be considered also in a continuum mechanics description.

As a first step, we concentrate on the simplest continuum setup without interphases, quantify the geometric effect, and compare it to the hybrid results. Our FE model consists of a cubic box with $L^{M D}=21.3 \mathrm{~nm}$ edge length for the polymer matrix, coinciding with the dimension of the particle domain in the hybrid simulation, which is confined by the anchor points. The polymer is treated geometrically nonlinear, i.e. with a large strain formulation. Furthermore, it is described by linear elasticity with a Young's modulus $E=1.5 \mathrm{GPa}$ and a Poisson's ratio $\nu=0.4$. These values do not coincide with the coarse grained MD polymer, still, the differences do not affect the resulting inhomogeneous strain distribution in a relevant manner, but only the stress level, which is not considered here. For the NPs, two spheres are embedded into the matrix at the same initial distances as used in the hybrid simulations. On the constitutive side, the NPs are described like the matrix, but with $E=88.7 \mathrm{GPa}$ and $\nu=0.23$, which are values commonly used for bulk silica. All components of the model are discretized by tetrahedral elements with quadratic shape functions, the interfaces between matrix and particles are realized as a conforming mesh, i.e. without any additional contact description. Loading is applied via Dirichlet boundary conditions identical to the hybrid simulations. Geometric quantities as given above are used.

From Figure 7 it is evident that also the normalized strain $\tilde{\varepsilon}^{N P}$ between NP centers in the FE simulation depends significantly on the initial interparticle distance $L^{N P}$ (similar to the hybrid simulation), but not on the overall strain $\varepsilon^{M D}$ (different to the hybrid simulation). In case of the closest NP configuration with $L^{N P}=5.93 \mathrm{~nm}$, one obtains $\tilde{\varepsilon}^{N P}=0.86$ for $\varepsilon^{M D}=0.41 \%$, which slightly increases to $\tilde{\varepsilon}^{N P}=0.87$ for $\varepsilon^{M D}=7.75 \%$. The system with $L^{N P}=9.47 \mathrm{~nm}$ renders $\tilde{\varepsilon}^{N P}=0.99$ and only a very small increase in the range of $10^{-3}$ can be observed. 


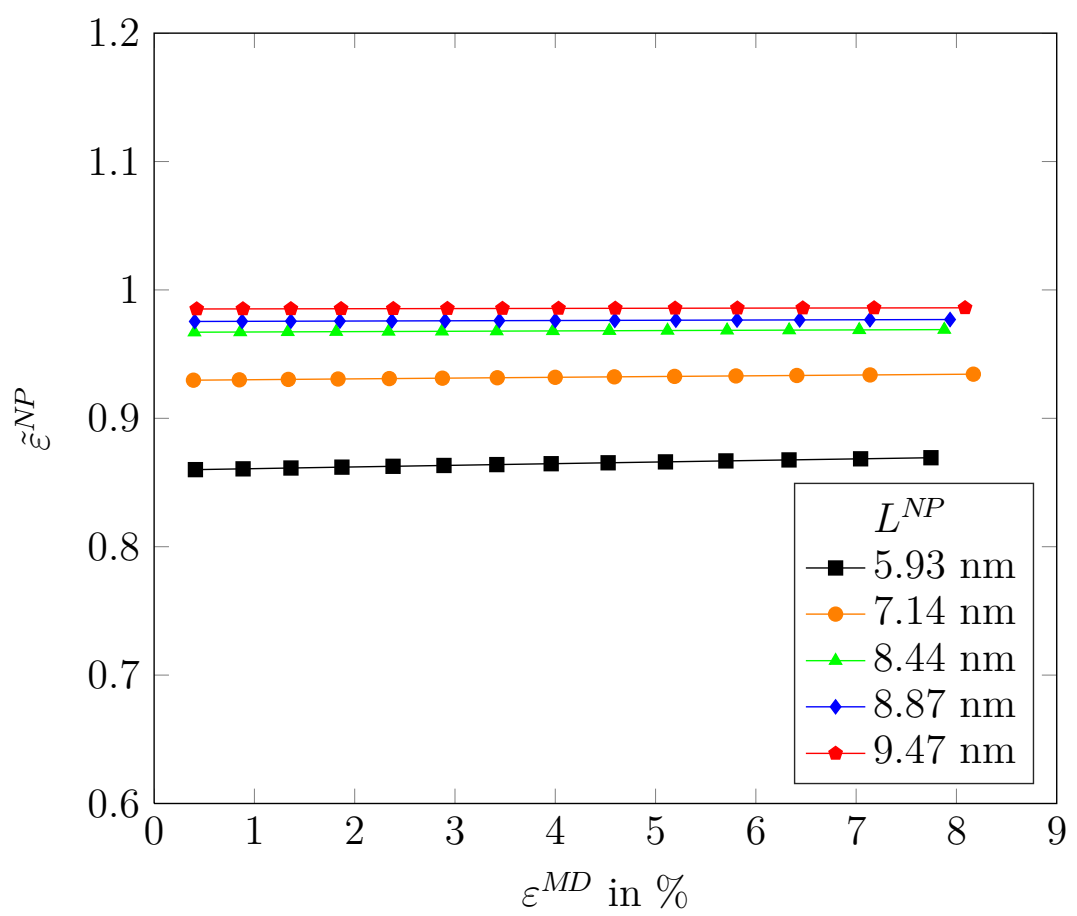

FIG. 7. Pure FE simulation: normalized interparticle strain $\tilde{\varepsilon}^{N P}$ as a function of the global strain $\varepsilon^{M D}$ for various initial interparticle distances $L^{N P}$

\section{Hybrid MD-FE vs. pure FE system: conclusions}

Based on the observations of the preceding sections, we discuss in the following the dependencies of $\tilde{\varepsilon}^{N P}$ on the integral MD strain $\varepsilon^{M D}$ and on the initial NP distance $L^{N P}$.

1. $\tilde{\varepsilon}^{N P}$ as a function of $\varepsilon^{M D}$

In the hybrid system, $\tilde{\varepsilon}^{N P}$ increases for increasing $\varepsilon^{M D}$, whereby it is in the range of 0.6 to 1.1 (for $L^{N P}>5.93 \mathrm{~nm}$ ) and of 0.3 to 1.0 (for $L^{N P}=5.93 \mathrm{~nm}$ ), see Figure 6. All curves show fluctuations within a range of approximately 0.2 which, however, do not affect the qualitative findings, but leave a clear trend. In the pure FE system, $\tilde{\varepsilon}^{N P}$ shows almost no dependency on $\varepsilon^{M D}$ and is in the range of 0.85 to 1.0 for all $L^{N P}$, see Figure 7 . Thus, both systems render results in the same range for large $\varepsilon^{M D}$, whereas the values of the hybrid system are significantly lower in case of small $\varepsilon^{M D}$. The largest deviation is obtained for the smallest NP distance. 


\section{2. $\tilde{\varepsilon}^{N P}$ as a function of $L^{N P}$}

In case of the hybrid system, $\tilde{\varepsilon}^{N P}$ takes its smallest values for the smallest NP distance, whereby all curves are in the same range for $L^{N P}>5.93 \mathrm{~nm}$. In contrast, a clear dependency for all NP distances is obtained in the pure FE system. Here, $\tilde{\varepsilon}^{N P}$ increases with increasing NP distance, whereby values of $\tilde{\varepsilon}^{N P}$ for large NP distances are closer together. As in the hybrid case, the values of $\tilde{\varepsilon}^{N P}$ for the shortest NP distance are significantly lower. Hence, the hybrid and the pure FE system lead to qualitatively similar results, although the quantitative values differ. The observation that the NPs do not follow the overall strain may hence be explained by the geometric situation, at least partially.

\section{Evaluation}

The above findings have to be evaluated carefully. Beyond geometric effects in a continuum mechanics sense as discussed above, one might argue that differences between the hybrid and the pure FE solution originate solely from fluctuations of quantities employed in eqs. (1) - (4) at particle resolution. In fact, we obtain significant deviations especially at small strains and small NP distances. In particular, $\tilde{\varepsilon}^{N P}$ is more sensitive to deviations when its denominator and thus the NP distances are small. Second, deviations may occur more likely in case of small strains due to small changes in length. However, even if this leads to pronounced deviations of $\tilde{\varepsilon}^{N P}$ at small strains and small NP distances, it does not alter the qualitative behavior of the systems. To study such effects in detail, more extended investigations have to be carried out.

The clear dependency of $\tilde{\varepsilon}^{N P}$ on $\varepsilon^{M D}$ in case of the hybrid system is a qualitative difference between hybrid and pure FE simulations that can be explained neither by geometric effects nor by fluctuations. Instead, it is plausible that spatially varying mechanical properties in the polymer form interphases around the NPs which cannot be accounted for in simple continuum mechanics descriptions. A more sophisticated continuum setup would be required which explicitly includes interphases, i.e. material parameter gradients around the NPs. Figure 8 displays the normalized strain $\tilde{\varepsilon}^{N P}$ between NP centers computed in hybrid and pure FE simulations for the smallest and largest center-to-center distances $L^{N P}$. It is obvious that, in case of the smallest NP distance shown in Figure 8 a), $\tilde{\varepsilon}^{N P}$ is significantly lower 

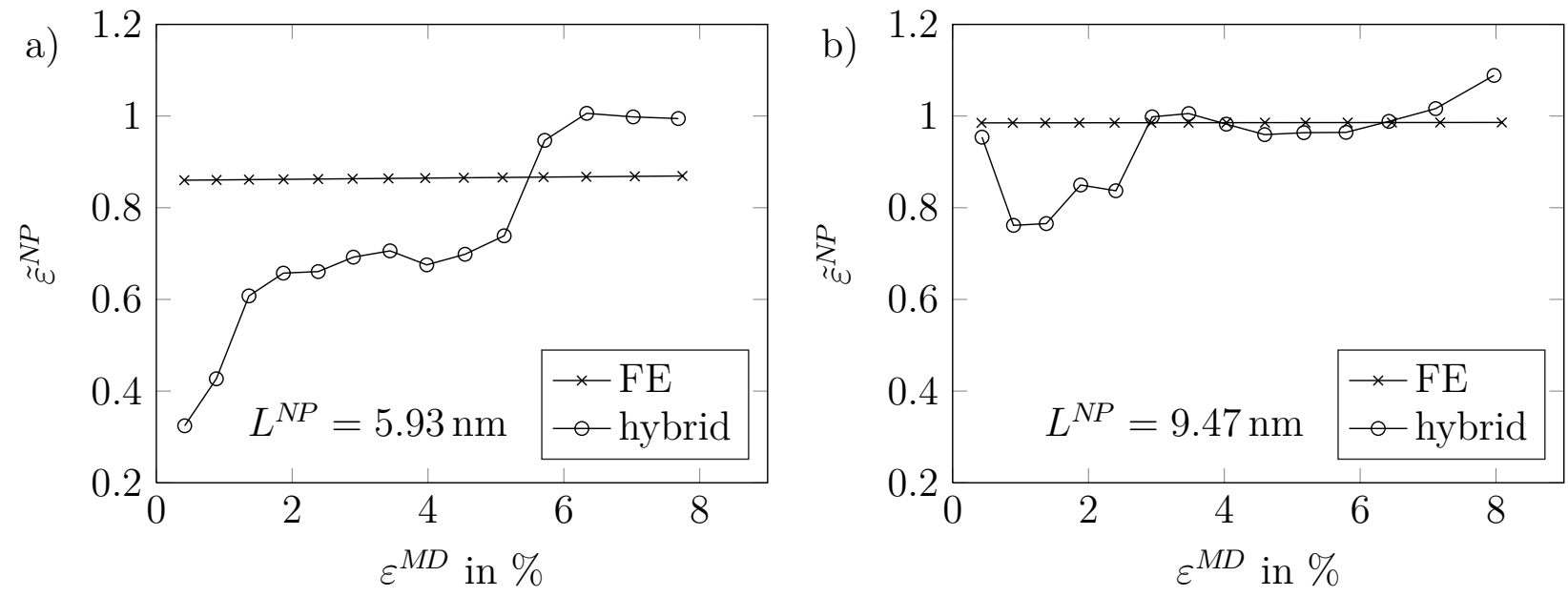

FIG. 8. Normalized strain between NP centers $\tilde{\varepsilon}^{N P}$ obtained from hybrid and pure FE simulations, a) for the closest center-to-center distance of NPs $\left(L^{N P}=5.93 \mathrm{~nm}\right)$ and b) for the largest NP distance $\left(L^{N P}=9.47 \mathrm{~nm}\right)$

than one would expect for a pure FE study. In consideration of an estimated interphase thickness of approximately $1 \mathrm{~nm}[8,31,33]$, this example is the only system where the two interphases show a sizeable overlap. Nevertheless, we want to mention that this value should be considered only as a rough estimate; see the detailed discussion in a recent work [8]. Some of our previous computer simulations [31] have also shown that the polymer in the interphase is stiffer than in the bulk, which makes it reasonable to expect less deformation there than in the softer bulk. On the other hand, the deviations between hybrid and pure FE results are nearly negligible in case of the largest NP distance, cf. Figure 8 b). This supports our assumption that the overlap of interphases leads to pronounced alterations of the mechanical response. However, statistically proven statements under the computational conditions given here are not yet possible.

Our results are in line with the aforementioned increased fragility of $\mathrm{NC}$ materials due to NP aggregation [48]. The fragility increases with increasing volume fraction of the NPs [48], which may be related to a higher probability of NP accumulations in case of higher NP loads. We are confident that future simulations of the here presented type will help to answer such questions at a microscopically profound basis. 
a)

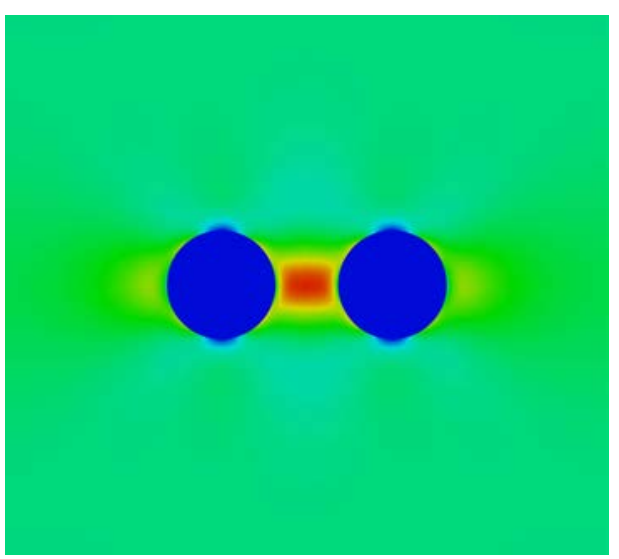

b)

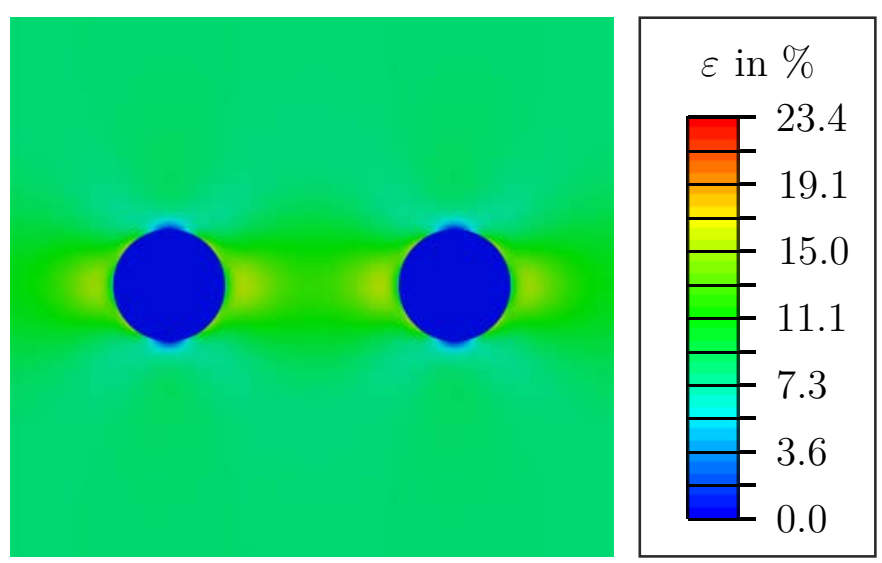

FIG. 9. FE-simulated distribution of local tensile strain $\varepsilon$ in the center plane at an overall strain $\varepsilon^{M D}=7.75 \%$, a) for minimum $(5.93 \mathrm{~nm})$ and b) maximum $(9.47 \mathrm{~nm})$ initial particle center-tocenter distances

\section{Continuative considerations}

Concluding this section, we give a short prospect of future activities based on our pure FE simulations. Figure 9 depicts the distribution of the local strain $\varepsilon$ in the tensile direction at maximum deformation for the two extremal values of interparticle distance $L^{N P}=5.93 \mathrm{~nm}$ and $L^{N P}=9.47 \mathrm{~nm}$. In both cases, as evident from Figure 8 , the normalized strain $\tilde{\varepsilon}^{N P}$ between the NP centers is smaller than one. In contrast, the local strain $\varepsilon$ in the region between the particles is significantly higher than the overall strain $\varepsilon^{M D}$, see Figure 9 . For the shortest NP distance, $\varepsilon$ is approximately three times higher than $\varepsilon^{M D}$, whereas this ratio is considerably lower for $L^{N P}=9.47 \mathrm{~nm}$. The fact that the local strain between the particles is that large in case of short NP distances is reasonable since the total change of length and thus the overall strain has to be distributed in the composite. If the filler particles are almost rigid, they are barely strained. The matrix, in turn, absorbs the total change of length, but based on a shorter initial distance. Consequently, the strain of the matrix is larger in the vicinity of the particles than elsewhere. If the NPs are very close, the change of length is based on a much shorter initial distance and the local strain significantly increases. Such highly strained polymer matrix regions most likely play an important role in the fragility of NCs containing NP agglomerates. A due description and evaluation of these regions that follows from the particle level and which is beyond the capability of classical 
FE considerations is currently under investigation.

A rough estimation of the interparticle polymer strain as computed in MD can be given when the NPs are considered to be rigid. This is surely a reasonable approximation since the NPs are much stiffer than the surrounding polymer matrix. The maximum strain $\varepsilon_{\max }^{M D}$ of the polymer matrix between the NP surfaces may then be estimated by

$$
\varepsilon_{\max }^{M D}=\frac{\ell^{N P}-L^{N P}}{L^{N P}-D^{N P}}=\frac{\ell^{N P}-L^{N P}}{L^{N P}} \frac{L^{N P}}{L^{N P}-D^{N P}}=\varepsilon^{N P} \frac{L^{N P}}{L^{N P}-D^{N P}}
$$

and the maximum normalized strain of the polymer matrix follows as

$$
\tilde{\varepsilon}_{\max }^{M D}=\frac{\varepsilon_{\max }^{M D}}{\varepsilon^{M D}}
$$

Then, in case of the closest NP distance and at $\varepsilon^{M D}=7.7 \%$, a normalized strain $\tilde{\varepsilon}_{\max }^{M D}=$ 3.04 is obtained from the hybrid method, whereas it is only $\tilde{\varepsilon}_{\max }^{M D}=1.88$ for the maximum interparticle distance. From the FE simulation as given in Figure 9, a maximum strain of approximately $23 \%$ is derived for the smallest NP distance, which yields $\tilde{\varepsilon}_{\max }^{M D}=2.96$. For the largest NP distance, the maximum strain is about $15 \%$ and thus $\tilde{\varepsilon}_{\max }^{M D}=1.94$. For both NP distances, the values of the hybrid and the pure FE system are very close together, although they represent only a rough estimation. Thus, we are convinced that more sophisticated studies based on the presented hybrid simulation approach under consideration of effects that can be captured only by particle-based considerations will help for a better understanding of the complex processes taking place in polymer nanocomposites.

At present, however, the hybrid method is not yet able to capture phenomena as e.g. large strains, inelasticity, or fracture. First steps in these directions are currently being developed, departing from an exact mechanical analysis of the particle-based polymer system and its supposed inelastic properties. Furthermore, a profound investigation of the interphases and their specific constitutive relations is required, in particular for systems with local agglomerations at low nanoparticle densities. In this process, our hybrid method will play an important role since large systems with agglomerating nanoparticles are barely accessible by pure MD approaches. With such a hybrid tool at hand, we expect that effects originating from the atomistic level can be incorporated into continuum mechanics systems at length and time scales relevant in engineering and mechanical testing. 


\section{SUMMARY}

The mechanical behavior of silica-polystyrene nanocomposites has been studied by using a hybrid simulation technique. Our method couples a particle domain to a continuum domain modeled by an FE approach. The particle-based part of our approach has been performed by molecular dynamics simulations at a coarse grained scale. A uniaxial deformation has been applied to the system by prescribing a displacement in two opposite directions on the outermost finite element boundaries. We have simulated nanocomposites with one and two silica NPs embedded in atactic glassy polystyrene. The results for the system with one NP have been compared to those obtained previously from a pure PS system. Thereby, we have found that our hybrid method is able to capture the influence of the NP properly. In a next, step we have calculated the orientation of polymer segments with respect to the deformation direction. The polymer orientation relative to the NP surface has not been discussed in the present work. For recent studies of this quantity in the absence of a deformation see [33]. For small strains, the polymer segments keep their orientation. However, in case of larger strains they rotate and start to align with the deformation direction.

For the system with two NPs we have calculated the total strain $\varepsilon^{M D}$ in the MD domain and the integral strain $\varepsilon^{N P}$ measuring the change in distance between the NP centers. The ratio between the two strain values, introduced as the normalized strain $\tilde{\varepsilon}^{N P}$, has been investigated for different NP distances. According to our calculations, this ratio is a function of the strain applied to the system and of the initial distance between the NP centers. We have found that the two NPs do not displace with the overall strain, especially in case of small strains and initial NP distances. These results have been compared to those obtained from a pure FE study of the same NC configuration. We could show that a part of the observed strain inhomogeneities may be captured by a rather simple continuum description. However, one has to consider spatially varying mechanical properties of the polymer in the vicinity of the NPs, i. e. interphases, to completely explain the observed behavior. Such effects occurring in the interphase between polymer matrix and NPs are inherently captured only by the particle-based approach. They can be reproduced, e.g., if material parameter gradients are included in the continuum mechanics description which in turn might be obtained from physical experiments or particle simulations.

The inhomogeneous strain distribution in NC systems shown here might help to explain 
why nanocomposites are more fragile than the bulk polymer and where a break might start. This is a very important question in the nanocomposite field and - to the best of our knowledge - has not been answered up to now. Finding the answer of this question might support the design of novel materials. Our hybrid MD-FE method hence has to be extended to geometrical and physical nonlinearities to allow for studying systems under large deformations and up to failure.

\section{ACKNOWLEDGMENTS}

Funding by the European Union within the collaborative "Nanomodel" project (No. 211778) is gratefully acknowledged as well as by the German Research Foundation (DFG) within the DFG-priority programme 1369 "Polymer-Solid Contacts: Interfaces and Interphases" and through the Cluster of Excellence Engineering of Advanced Materials (research area A3).

[1] S. K. Kumar and R. Krishnamoorti, Annual Review of Chemical and Biomolecular Engineering 1, 37 (2010).

[2] L. Schadler, Nature Materials 6, 257 (2007).

[3] K. Johnston and V. Harmandaris, Macromolecules 46, 5741 (2013), http://dx.doi.org/10.1021/ma400357r.

[4] Y. Li, M. Kröger, and W. K. Liu, Soft Matter 10, 1723 (2014).

[5] V. Ganesan and A. Jayaraman, Soft Matter 10, 13 (2014).

[6] R. Richert, Annual Review of Physical Chemistry 62, 65 (2011), pMID: 21090966, http://dx.doi.org/10.1146/annurev-physchem-032210-103343.

[7] S. Kim and J. M. Torkelson, Macromolecules 44, 4546 (2011), http://dx.doi.org/10.1021/ma200617j.

[8] E. Voyiatzis, M. Rahimi, F. Müller-Plathe, and M. C. Böhm, Macromolecules 47, 7878 (2014), http://dx.doi.org/10.1021/ma500556q.

[9] G. Allegra, G. Raos, and M. Vacatello, Progress in Polymer Science 33, 683 (2008).

[10] J. Liu, L. Zhang, D. Cao, and W. Wang, Physical Chemistry Chemical Physics 11, 11365 
(2009).

[11] Q. Zeng, A. Yu, and G. Lu, Progress in Polymer Science 33, 191 (2008).

[12] J. J. de Pablo, Annual Review of Physical Chemistry 62, 555 (2011).

[13] J. Bouvard, D. Ward, D. Hossain, S. Nouranian, E. Marin, and M. Horstemeyer, Journal of Engineering Materials and Technology 131, 041206 (2009).

[14] T. Karakasidis and C. Charitidis, Materials Science and Engineering: C 27, 1082 (2007), \{EMRS\} 2006 Symposium A: Current Trends in Nanoscience - from Materials to Applications.

[15] C. Peter and K. Kremer, Soft Matter 5, 4357 (2009).

[16] F. Müller-Plathe, ChemPhysChem 3, 754 (2002).

[17] R. A. Riggleman, G. Toepperwein, G. J. Papakonstantopoulos, J.-L. Barrat, and J. J. de Pablo, The Journal of Chemical Physics 130, 244903 (2009).

[18] A. Villa, C. Peter, and N. F. van der Vegt, Physical Chemistry Chemical Physics 11, 2077 (2009).

[19] S. Fritsch, S. Poblete, C. Junghans, G. Ciccotti, L. Delle Site, and K. Kremer, Physical Review Letters 108, 170602 (2012).

[20] T. Werder, J. H. Walther, and P. Koumoutsakos, Journal of Computational Physics 205, 373 (2005).

[21] P. Koumoutsakos, Annual Review of Fluid Mechanics 37, 457 (2005).

[22] G. Csányi, T. Albaret, M. C. Payne, and A. De Vita, Physical Review Letters 93, 175503 (2004).

[23] J. Rottler, S. Barsky, and M. O. Robbins, Physical Review Letters 89, 148304 (2002).

[24] S. Qi, H. Behringer, and F. Schmid, New Journal of Physics 15, 125009 (2013).

[25] D. Davydov, J.-P. Pelteret, and P. Steinmann, Computer Methods in Applied Mechanics and Engineering 277, 260 (2014).

[26] R. Delgado-Buscalioni, K. Kremer, and M. Praprotnik, The Journal of Chemical Physics 131, 244107 (2009).

[27] S. Pfaller, M. Rahimi, G. Possart, P. Steinmann, F. Müller-Plathe, and M. Böhm, Computer Methods in Applied Mechanics and Engineering 260, 109 (2013).

[28] M. Rahimi, H. A. Karimi-Varzaneh, M. C. Böhm, F. Müller-Plathe, S. Pfaller, G. Possart, and P. Steinmann, Journal of Chemical Physics 134, 154108 (2011).

[29] T. Belytschko, R. Gracie, and G. Ventura, Modelling and Simulation in Materials Science 
and Engineering 17, 043001 (2009).

[30] P. Moseley, J. Oswald, and T. Belytschko, International Journal for Numerical Methods in Engineering 92, 835 (2012).

[31] M. Rahimi, I. Iriarte-Carretero, A. Ghanbari, M. C. Böhm, and F. Müller-Plathe, Nanotechnology 23, 305702 (2012).

[32] A. Ghanbari, T. V. M. Ndoro, F. Leroy, M. Rahimi, M. C. Böhm, and F. Müller-Plathe, Macromolecules 45, 572 (2012), http://pubs.acs.org/doi/pdf/10.1021/ma202044e.

[33] T. V. M. Ndoro, E. Voyiatzis, A. Ghanbari, D. N. Theodorou, M. C. Böhm, and F. MüllerPlathe, Macromolecules 44, 2316 (2011), http://dx.doi.org/10.1021/ma102833u.

[34] A. Ghanbari, M. Rahimi, and J. Dehghany, The Journal of Physical Chemistry C 117, 25069 (2013), http://pubs.acs.org/doi/pdf/10.1021/jp407109r.

[35] P. J. Hoogerbrugge and J. M. V. A. Koelman, Europhysics Letters 19, 155 (1992).

[36] R. D. Groot and P. B. Warren, The Journal of Chemical Physics 107, 4423 (1997).

[37] H. Ben Dhia and G. Rateau, International Journal for Numerical Methods in Engineering 62, $1442(2005)$.

[38] S. Pfaller, G. Possart, P. Steinmann, M. Rahimi, F. Müller-Plathe, and M. C. Böhm, Computational Mechanics 49, 565 (2012).

[39] S. Liu, A. Gerisch, M. Rahimi, J. Lang, M. C. Böhm, and F. Müller-Plathe, The Journal of Chemical Physics 142, 104105 (2015).

[40] H. Qian, P. Carbone, X. Chen, H. A. Karimi-Varzaneh, and F. Müller-Plathe, Macromolecules 41, 9919 (2008).

[41] D. Reith, M. Pütz, and F. Müller-Plathe, Journal of Computational Chemistry 24, 1624 (2003).

[42] W. L. Jorgensen, D. S. Maxwell, and J. Tirado-Rives, Journal of the American Chemical Society 118, 11225 (1996), http://dx.doi.org/10.1021/ja9621760.

[43] P. E. M. Lopes, V. Murashov, M. Tazi, E. Demchuk, and A. D. MacKerell Jr., The Journal of Physical Chemistry B 110, 2782 (2006), pMID: 16471886, http://dx.doi.org/10.1021/jp055341j.

[44] M. P. Allen and D. J. Tildesley, Computer simulation of liquids (Oxford university press, 1989).

[45] M. Langeloth, T. Sugii, M. C. Böhm, and F. Müller-Plathe, The Journal of Chemical Physics 
143, 243158 (2015), http://dx.doi.org/10.1063/1.4937627.

[46] S. Pfaller, Multiscale Simulation of Polymers - Coupling of Continuum Mechanics and Particle-Based Modelling, Ph.D. thesis, Friedrich-Alexander-Universität Erlangen-Nürnberg (2015).

[47] H. Eslami, M. Rahimi, and F. Müller-Plathe, Macromolecules 46, 8680 (2013), http://dx.doi.org/10.1021/ma401443v.

[48] E. Kontou and G. Anthoulis, Journal of Applied Polymer Science 105, 1723 (2007).

[49] B. A. P. Betancourt, J. F. Douglas, and F. W. Starr, Soft Matter 9, 241 (2013).

[50] J. Jancar, J. Douglas, F. Starr, S. Kumar, P. Cassagnau, A. Lesser, S. Sternstein, and M. Buehler, Polymer 51, 3321 (2010).

[51] G. D. Smith and D. Bedrov, Langmuir 25, 11239 (2009), pMID: 19788206, http://dx.doi.org/10.1021/la902329v. 